\title{
Growth of linear charged micelles
}

\author{
Theo Odijk \\ Department of Polymer Technology, Faculty of Chemical Engineering and Materials Science, Delft University of Technology, \\ P.O. Box 5045, 2600 GA Delft (Netherlands)
}

\begin{abstract}
The electrostatics of micellar growth is reviewed and extended for solutions containing excess salt. In dilute solution the expansion of a linear micelle with increasing salt concentration is explained for a wide range of ionic strength. When the micellar charge density is very high, counterions condense nonuniformly onto the micellar rod. In that case the micelle may contract upon the addition of salt. In semidilute solutions the excluded-volume effect is an additional factor complicating the ionic strength dependence of micellar growth.
\end{abstract}

Keywords: Linear charged micelles; Micellar growth; Polyelectrolytes

\section{Introduction}

Invariably, highly simplified models have been employed to describe polyelectrolytes. The polyions have often been viewed as charged lines, cylinders or worms and the solvent as structureless. In reality of course the backbone is highly structured, the charges are discrete and the polar solvent responds nonuniformly to the presence of the polyelectrolyte. It is reasonable to suppose that continuum models become realistic in the limit of low ionic strength when the Debye screening length is so large that long-range electrostatics dominates all other interactions. Yet, rather surprisingly, polyelectrolyte theories disregarding structure are quite successful even at intermediate salt concentration. The merits and deficiencies of current modeling have been admirably reviewed by Michel Mandel [1]. Polyelectrolytes in excess salt are fairly well understood although a microscopic justification of continuum theories is lacking. By contrast, salt-free polyelectrolyte solutions remain an enigma: all current models neglect the strong coupling of the small-ion distribution to the semidilute polyion structure.

It is tempting to extrapolate the reasoning adopted for polyelectrolytes to the case of slender linear micelles that are charged. Thus we model each micelle as a smooth cylinder hearing hemispherical caps [2]. The uniform surface charge density of the cylindrical body may differ from that of the two caps. The complete neglect of the internal surfactant structure is, without doubt, a drastic assumption. The charge density is often thought to be determined by the balance of in the main hydrophobic versus electrostatic forces [2-4]. However, it is becoming increasingly clear that this picture is oversimplified [5]. In the absence of a quantitative theory this author naively assumes the surface charge density is invariant, for instance independent of the ionic strength. This supposition is not as bad as it seems. First, at low salt concentrations the electrostatic free energy of a micellar rod depends only slightly, that is logarithmically, on the ionic strength. In addition, if the charge density is high, the effective charge 
density remains more or less fixed because counterions condense onto the rod.

In a solution containing aggregates of different composition, the thermodynamic quantity of interest is the chemical potential of one amphiphile. The growth of micellar rods is then an end effect [3]. To set up an electrostatic theory of growth we have to go beyond the usual extensive expression for the free energy which is proportional to the micellar length and hence equivalent to standard polyelectrolyte theory. In this review end effects of electrostatic origin under excess salt conditions will be summarized. The connection between end effects and micellar growth is first established for uncharged micelles.

\section{Uncharged linear micelles}

The phenomenological ideas concerning the growth of uncharged micellar rods are fairly well established although there is no consensus on detail. The chemical potential $\mu(s)$ of a rodlike aggregates consisting of $s$ amphiphiles is written as [3]

$\mu(s)=s \mu_{0}+K+\log X(s)-n \log s$

$s \geq s_{0}$

All the energy terms are scaled with respect to $k_{\mathrm{B}} T$ where $T$ is the thermodynamic temperature of the system and $k_{\mathrm{B}}$ is Boltzmann's constant. The amphiphiles in the cylindrical body have a chemical potential $\mu_{0}$ but those in the two hemispherical caps have a higher potential for reasons left unspecified here. Hence, the reference chemical potential is split up into an extensive term $s \mu_{0}$ and an end cap term $K$ which is proportional to the number of surfactant molecules within the caps. We suppose $s$ is larger than some minimum aggregation number $s_{0}$ so that all the micellar aggregates are slender and rodlike. There is an ideal mixing term involving the mole fraction $X(s)$ of aggregates consisting of $s$ amphiphiles.

In ref. [3] $n$ was set equal to unity as arises from a consideration of the ideal mixing term. Later investigators [6,7] argued that the system loses translational and rotational degrees of free- dom on aggregation so that $n$ should equal six. Nevertheless, ascertaining the precise number of degrees of freedom in an aggregating system is no mean task witness the tortuous and unresolved route followed in other areas of physical chemistry (see for instance ref. [8] for a discussion on the formation of water droplets). Here, $n$ is supposed a free parameter pending further experimental work. In any event, in practice linear micelles are wormlike and semiflexibility would also change $n$.

One approximate way of dealing with eq. (1) is to neglect polydispersity entirely. One imposes the condition that the aggregates be monodisperse. The system is open at constant pressure and temperature so that the total Gibbs free energy has to be minimized. Since the total number of surfactant molecules is constant, one derives $s$ by minimizing $\mu(s) / s$, the chemical potential per amphiphile

$n \log s \approx K+\log X+n$

Here, the total mole fraction $X$ is identical with $X(s)$.

Actually, linear micelles are significantly polydisperse [3]. Equation (1) yields the distribution of aggregates since thermodynamic equilibrium requires that the chemical potential per amphiphile should be a constant $\bar{\mu}$.

$X(s)=s^{n} \mathrm{e}^{-p s-K}$

The constant $p \equiv \mu_{0}-\bar{\mu}$ is assumed to be positive otherwise the system would aggregate into infinite structures. Now the total mole fraction is also invariant

$X=\sum_{s} X(s) \approx \int_{s_{0}}^{\infty} \mathrm{d} s X(s)=\mathrm{e}^{-K} \int_{s_{0}}^{\infty} \mathrm{d} s s^{n} \mathrm{e}^{-p s}$

and it is to be expressed in terms of the average aggregation number $S$ via [3]

$S=\sum_{s} s X(s) / X \approx-\frac{\partial \log X}{\partial p}$

Equation (4) is approximated with the help of 
Laplace's method in which $X(s)$ is replaced by a Gaussian. Noting that $n \gg p s_{0}$, we have

$X \approx\left(\frac{2 \pi}{n}\right)^{1 / 2}\left(\frac{n}{p}\right)^{n+1} \mathrm{e}^{-K-n}$

Equation (5) then yields $S$ and on elimination of $p$ one obtains

$(n+1) \log S=K+n+c(n)+\log X$

The slowly varying function $c(n)$ is close to unity for all $n$. In effect, polydispersity does modify the growth of rodlike micelles (compare eq. 2 with eq. 7) but only quantitatively.

As expected the rods grow as the surfactant concentration increases, for entropic reasons. Furthermore they also lengthen as the cap energy $K$ is enhanced. This is plausible since the system tends to minimize energy by decreasing the number of micelles. In the subsequent analysis the form of eq. (7) will be addressed when electrostatic forces are present in a variety of circumstances.

\section{Micelles of low charge density at low ionic strength}

If the concentration $n_{\mathrm{s}}$ of monovalent salt is low, the Debye screening length is larger than the micellar diameter. One expects the detailed structure of the aggregate to become unimportant in this limit. A zero-order approximation is to replace the micellar rod by a finite line charge of length $L$ bearing $\nu$ elementary charges $q$ per unit length. The micelle is immersed in a solvent of permittivity $D$. In the Debye-Hückel approximation its electrostatic free energy is [9]

$f / k_{\mathrm{B}} T=\nu^{2} Q \int_{\epsilon}^{L} \mathrm{~d} t(L-t) t^{-1} \mathrm{e}^{-\kappa t}$

Here the relevant electrostatic scale is the Bjerrum length $Q \equiv \mathrm{q}^{2} / D k_{\mathrm{B}} T$ in terms of which the Debye length $\kappa^{-1}$ is given by $\kappa^{2}=8 \pi Q n_{\mathrm{s}}$. Equation (8) is deemed accurate when the micelle has a low charge density, i.e. when the charge density parameter $\bar{\xi} \equiv Q \nu$ is smaller than unity.

We focus on the limit where the cut-off $\epsilon$ is much smaller than the Debye radius which in turn is much smaller than the micellar length. Then the free energy $f$ can be split up into an extensive term $f_{0}$ proportional to $L$, that is proportional to the total number of surfactant molecules $s$, and an end effect $f_{1}$ given by [9]

$f_{1} / k_{\mathrm{B}} T=-\nu^{2} Q \kappa^{-1}$

Note that this expression bears a minus sign: a charge near one end experiences fewer interactions than a charge in the midsection. Moreover, $f_{1}$ is proportional to the Debye length because this is the approximate scale of nonuniformity at both ends of the micelle.

The electrostatic free energy (eq. 8) has to be added to the chemical potential (eq. 1). The growth constant $K$ can then be replaced by $K_{0}+f_{1} / k_{\mathrm{B}} T$ where $K_{0}$ is supposed independent of the ionic strength. In view of eqs. (7) and (9) we write [9]

$\log L=-\frac{\nu^{2} Q}{(n+1) \kappa}+$ constant

This predicts that the micelles should grow if salt is added to the solution.

Comparatively few micelles are rodlike at low ionic strength so it is hard to find systematic measurements of miccllar growth in this regime. Hoffmann et al. [10] performed small angle neutron scattering on cetylpyridinyl salicylate in $\mathrm{D}_{2} \mathrm{O}$ at low $\mathrm{NaCl}$ concentrations. The linear charge density of the rodlike micelles was asserted to be $\nu \approx 1 / 2 Q$. The constant $n$ is set equal to six in accordance with refs. [6] and [7]. Since we do not know the value of the constant in eq. (10), we specify it empirically by appealing to the experimental data at $0.5 \mathrm{~m} M \mathrm{NaCl}$. As shown in Table 1 , the theoretical predictions of the length at 2 and $8 \mathrm{~m} M$ coincide with those determined experimen-

Table 1

Length of cetylpyridinyl salicylate in $\mathrm{D}_{2} \mathrm{O}[10]$ versus the $\mathrm{NaCl}$ concentration as measured by small angle neutron scattering

\begin{tabular}{lll}
\hline$n_{\mathrm{s}}(\mathrm{m} M)$ & $\mathrm{L}(\mathrm{nm})$ & $\mathrm{L}_{\text {(theory) }}(\mathrm{nm})$ \\
\hline 0.5 & 55 & 55 \\
2 & 75 & 77 \\
8 & 100 & 85 \\
32 & 300 & 99 \\
\hline
\end{tabular}


tally to within the accuracy presented in ref. [10]. The large disparity between theory and experiment at $32 \mathrm{mM}$ is not surprising; the line approximation breaks down at short Debye lengths.

\section{Nonuniform counterion condensation}

If the charge density parameter $\bar{\xi}$ is greater than unity, the Debye-Hückel approximation as applied to the bare line charge becomes unrealistic. Incorporating nonlinear screening for finite lines or rods is complicated [11] so here the simple idea of counterion condensation [12] is applied to evaluate the end effect.

Suppose counterions have condensed onto a finite line charge. The effective charge density $\nu_{\text {eff }}(s)$ cannot be homogeneous but depends on the contour distance $s$ from one end. When the micelle is much longer than the Debye length, the dimensionless quantity $\xi(s) \equiv Q \nu_{\text {eff }}(s)$ should be set equal to unity [12] if $\vec{\xi}>1$, at least in the midsection. Our aim is to calculate $\xi(s)$ near the two ends, both regions of nonuniformity being a Debye length long.

Now in equilibrium the chemical potential $\mu_{i}$ (scaled by $k_{\mathrm{B}} T$ ) of a condensed counterion should be invariant along the micellar contour [13]

$\mu_{i}=\mu_{0}+\log (\bar{\xi}-\xi(s))-R \phi(s)=$ constant.

Here an ideal mixing entropy balances the electrostatic potential exerted on the counterion by the charged line. Actually, the micelle is not a line but a thin cylinder of radius $a$; in the limit $a \kappa \ll 1$, arguments advanced for lines [12] should remain asymptotically valid for cylinders [14]. The dimensionless potential can be expressed by

$\phi(s)=\int_{0}^{L} \mathrm{~d} t \frac{\xi(t) \exp -\kappa\left[(t-s)^{2}+a^{2}\right]^{1 / 2}}{\left[(t-s)^{2}+a^{2}\right]^{1 / 2}}$

At low ionic strength the Debye-Hückel approximation is quite reasonable again, provided we use the renormalized or effective charge density $\xi(t)[13]$.
In general the nonlinear integral equation (11) is very difficult to attack analytically. It so happens that it is fairly straightforward to find a solution [13] correct to the leading order when $L \kappa \gg 1,-\log \kappa a \gg 1$ and $\bar{\xi} \gg 1$ : the first two inequalities help simplify eq. (12); the third inequality allows us to neglect the ideal mixing contribution so that $\phi(s)$ is virtually independent of $s$ and equal to about $-2 \log \kappa a$ as for infinite lines [12] or thin infinite cylinders [14]. The leading term becomes

$$
\begin{array}{rlrl}
\xi(s) \approx \frac{2 \log \kappa a}{\log \kappa a+\log (a / s)} & & \text { for } a \leq s \leq \kappa^{-1} \\
& \approx 1 \quad \text { for } \kappa^{-1} \leq s \leq \frac{1}{2} L
\end{array}
$$

$\xi(s)=\xi(L-s)$

Hence, relatively fewer counterions condense on the ends than on the midsection.

Under the conditions specified above, the mixing entropy is negligible in comparison with the free energy $f_{\mathrm{c}}$ obtained by charging a bare line up to a charge density $\xi(s)$

$$
\begin{aligned}
f_{\mathrm{c}} / k_{\mathrm{B}} T & \approx \frac{1}{2} Q^{-1} \int_{a}^{L-a} \mathrm{~d} s \xi(s) \phi(s) \\
& \approx-Q^{-1} \log \kappa a \int_{a}^{L-a} \mathrm{~d} s \xi(s)
\end{aligned}
$$

Accordingly, the nonextensive end contribution is given by [13]

$$
\begin{aligned}
f_{1} / k_{\mathrm{B}} T & \approx-2 Q^{-1} \log \kappa a \int_{a}^{\kappa^{-1}} \mathrm{~d} s(\xi(s)-1) \\
& \approx 1 / \kappa Q
\end{aligned}
$$

where the integral has been expanded asymptotically to leading order. Note that in eqs. (13-15) the cut-off $a$ has to be introduced in order to conform to eq. (12) which converges for nonzero a.

Equations (1), (7) and (15) lead to an anomalous growth law [13]

$(n+1) \log L=\mathrm{constant}+1 / \kappa Q$

The effect of counterions condensing nonuniformly is remarkable: it changes the sign of eq. (10). This comes about because the effective elec- 
trostatic potential does not change along the micellar contour. Therefore, there are fewer counterions condensing on the two ends so that the effective charge density is lower along the midsection. As yet no experiments seem to have been carried out in the regime discussed here.

\section{Highly charged micelles at intermediate ionic strength}

In the previous sections attention was focused on the limit of low ionic strength which should be reasonably universal. A nonuniversal limit amenable to analysis is the thin double layer approximation: $a \kappa>1$. We also require the surface charge to be continuous so the ionic strength should not be too high: the Debye radius must be larger than the typical distance between neighbouring charges on the micellar surface. In practice, this regime of intermediate ionic strength is in between about 0.3 and $0.02 M$.

Under these circumstances the electrostatic free energy of two hemispherical caps of radius $a_{\mathrm{s}}$ can be calculated in the Poisson-Boltzmann approximation. At high surface charge density $\sigma_{\mathrm{s}}$ it reduces to [4]

$f_{\mathrm{s}, \mathrm{el}} / k_{\mathrm{B}} T \approx-\frac{8 a_{\mathrm{s}}}{Q} \log \pi Q \sigma_{\mathrm{s}} \kappa^{-1}$

If the same charges were uniformly arranged on the surface of a cylindrical body of radius a and charge density $\sigma_{c}$, their electrostatic free energy would be [4]

$f_{\mathrm{c}, \mathrm{el}} / k_{\mathrm{B}} T \approx-\frac{4 a_{\mathrm{s}}^{2} \sigma_{\mathrm{s}}}{Q a \sigma_{\mathrm{c}}} \log \pi Q \sigma_{\mathrm{c}} \kappa^{-1}$

Equations (7), (17) and (18) yield

$\log L=\mathrm{constant}+h \log n_{\mathrm{s}}$

where the nonuniversal constant

$h=\frac{2 a}{Q(n+1)}\left[2-\frac{a_{\mathrm{s}} \sigma_{\mathrm{s}}}{a \sigma_{\mathrm{c}}}\right]$

is expected to be positive.

Again, the micellar rods lengthen with increasing concentration. This effect is not difficult to understand. The absolute electrostatic potential at the surface of a sphere is less than that at a cylindrical surface of similar charge density. Furthermore, the difference between the respective electrostatic interactions decreases as the screening is enhanced.

The growth of linear micelles has been studied extensively at intermediate ionic strengths. For instance Ikeda et al. [15-18] found that a variety of micelles conformed to eq. (19) with $h$ varying from about 1 to 4 . Theoretically, $h$ would have a similar range of values. Thus the agreement seems satisfactory given the present uncertainty in $n$ and the surface parameters.

\section{Excluded-volume effect}

Slender micelles interact strongly at semidilute volume fractions. In that case excluded-volume terms have to be added to eq. (1). In the following discussion we temporarily let the micelles by monodisperse.

At fairly low concentrations binary collisions between the micellar rods predominate so we focus attention on the second virial coefficient only

$B_{2}=\frac{1}{2}\left\langle\int \mathrm{d} \boldsymbol{R}\left(1-\mathrm{e}^{-U / k_{\mathrm{B}} T}\right)\right\rangle$

This is an orientational average of a cluster integral depending on the energy $U(\boldsymbol{R}, \sin \gamma)$ between two micelles skewed at an angle $\gamma$ and whose centers are separated by a vector distance $\boldsymbol{R}$.

We first analyze $B_{2}$ for long micelles in the Debye-Hückel approximation $(\bar{\xi} \leq 1, L \kappa \gg 1$ and $a \kappa \ll 1$ ). The zero-order approximation to eq. (20) is obtained by supposing the ionic cloud enveloping a micelle is homogeneous i.e. equivalent to that surrounding an infinite line charge. In addition, any end effects are deleted. We then have a well-known result [19] in terms of an effective diameter.

$$
\begin{aligned}
B_{2,0} & \approx \frac{\pi}{4} L^{2} \kappa^{-1} \log 2 \pi Q \nu^{2} \kappa^{-1} \\
& \equiv \frac{\pi}{4} L^{2} D_{\mathrm{eff}}
\end{aligned}
$$


There are two first-order corrections to this expression [20]. First of all the ionic cloud extends beyond each micellar axis. Hence, each charged line bears two "hemispherical" caps, so to speak, which signify the range of the electrostatic potential. This yields a positive end contribution to the second virial coefficient [20].

$B_{2,+} \approx \pi L \kappa^{-2} \log ^{2} \pi Q \nu^{2} \kappa^{-1}$

On the other hand, as discussed in Section 3, the ionic cloud is nonuniform near the micellar ends, the electrostatic potential being less than supposed in the zero-order approximation. This amounts to a negative end effect [20].

$B_{2,-} \approx-L \kappa^{-2} \log ^{1 / 2} \pi Q \nu^{2} \kappa^{-1}$

which is overwhelmed by the other end term eq. (22). In conclusion the second virial coefficient may be written as

$B_{2} \approx \frac{\pi}{4} L^{2} D_{\mathrm{eff}}+\pi L D_{\mathrm{eff}}^{2}$

Note that the effective diameter is also the relevant scale in the end contribution.

Equation (24) is too restrictive for the micelles are not monodisperse. The second virial coefficient pertaining to the interaction between two aggregates consisting of respectively $r$ and $t$ amphiphiles is a straightforward extension of eq. (24) [24]

$B_{r t}=\frac{\pi}{4} L_{r} L_{t} D_{\mathrm{eff}}+\frac{\pi}{2}\left(L_{r}+L_{t}\right) D_{\mathrm{eff}}^{2}$

If there are $N_{r} r$-micelles and so forth in a volume $V$, the excluded-volume contribution to the total Helmholtz free energy is

$\Delta F=\sum_{r} \sum_{r} B_{r t} N_{r} N_{t} / V$

The micellar interactions cause the chemical potential of an $s$-micelle to increase by an amount

$$
\begin{aligned}
\Delta \mu_{s}= & \frac{\partial \Delta F}{\partial N_{s}}=2 \sum_{t} B_{s t} N_{t} / V \\
= & \frac{2 L_{s} D_{\text {eff }} \phi}{D^{2}}+\frac{\pi L_{s} D_{\text {eff }}^{2} N_{0}}{V} \sum_{s} \frac{X(s)}{S} \\
& +\frac{4 D_{\text {eff }}^{2} \phi}{D^{2}}
\end{aligned}
$$

Here, $D=2 a$ is the micellar diameter, $N_{0}$ is the number of solvent molecules and $\phi \equiv$ $\sum_{s} \pi N_{s} L_{s} D^{2} / 4 V$ represents the surfactant volume fraction. The effective diameter $D_{\text {eff }}$ may be generalized [20] so that $D_{\text {eff }} \rightarrow D$ at high salt.

In eq. (27) there are two extensive terms proportional to $s$ which do not influence the micellar growth. An analysis along the lines of eqs. (1)-(6) ultimately yields

$$
\begin{gathered}
(n+1) \log L=\text { constant }+\log \phi+4\left(\frac{D_{\text {eff }}}{D}\right)^{2} \phi \\
-Q \nu^{2} \kappa^{-1} \quad(\bar{\xi} \leq 1)
\end{gathered}
$$

A similar expression was presented in ref. (20) but with different numerical coefficients owing to the monodisperse constraint on the micellar growth. On adding a simple salt to the solution, the electrostatic excluded volume decreases. At low ionic strength this effect may offset the growth arising from the change in the self energy (the last term in eq. (28)). I am not aware of any experimental data in the regime pertaining to eq. (28).

When the micelles are very highly charged $(\bar{\xi}$ $\gg 1$ ), we may replace the bare charge in eq. (24) by an effective one and set $\xi \equiv Q \nu_{\text {eff }}$ equal to unity to a first approximation. In view of the counterion condensation being nonuniform, eq. (23) may well reverse its sign but it is overwhelmed by the other and effect (eq. 22). Accordingly, we have

$$
\begin{aligned}
(n+1) \log L= & \text { constant }+\log \phi \\
& +\frac{4 \phi \log ^{2}(2 \pi / Q \kappa)}{\kappa^{2} D^{2}}+\frac{1}{Q \kappa} \\
(\bar{\xi} \gg 1) &
\end{aligned}
$$

Under these conditions, the micelles should always decrease in length upon addition of salt to the solution.

\section{Concluding remarks}

At low ionic strength in excess salt the behavior of slender rodlike micelles should be complex and intriguing. Upon increasing the salt concentration the micelles may expand or contract depending on 
the charge density or volume fraction. Only a small number of experimental investigations has been carried out at low salt, possibly because few linear micelles exist under these circumstances. It is hoped that the present review will stimulate research in the regimes emphasized here.

Micellar growth in salt-free solutions is also an open problem in spite of several preliminary studies [21-23]. As stressed in the Introduction, the structure of salt-free polyelectrolytes is unresolved even when the polyions are rodlike. It seems to me that in that case the end effects are a great deal subtler than the ones surveyed in this review.

\section{Acknowledgment}

It is a pleasure to thank Prof. Michel Mandel for introducing the author to polyelectrolytes many years ago and for the vast number of exhilirating discussions spanning two decades on a variety of topics in physical chemistry.

\section{References}

1 M. Mandel, Polyelectrolytes, in: Encyclopaedia of Polymer Science and Engineering, 2nd edn. (Wiley, New York, NY, 1988).

2 J.N. Israelachvili, Intermolecular and Surface Forces (Academic Press, San Diego, CA, 1987).
3 J.N. Israelachvili, D.J. Mitchell and B.W. Ninham, J. Chem. Soc. Faraday Trans. 72 (2) (1976) 1525.

4 D.J. Mitchell and B.W. Ninham, J. Phys. Chem. 87 (1983) 2996.

5 A. Ben-Shaul and W.M. Gelbart, Ann. Rev. Phys. Chem. 36 (1985) 179.

6 R. Nagarajan and E. Ruckenstein, I. Colloid Interface Sci. 71 (1979) 580.

7 W.E. McMullen, W.M. Gelbart and A. Ben-Shaul, J. Phys. Chem. 88 (1984) 6649.

8 H.R. Pruppacher and J.D. Klett, Microphysics of Clouds and Precipitation (Reidel, Dordrecht, 1978) Chapter 7.

9 T. Odijk, J. Phys. Chem. 93 (1989) 3888.

$10 \mathrm{H}$. Hoffmann, J. Kalus, H. Thurn and K. Ibel, Ber. Bunsen Ges. Phys. Chem. 87 (1983) 1120.

11 G.V. Ramanathan and C.P. Woodbury, J. Chem. Phys. 77 (1982) 4133.

12 G.S. Manning, J. Chem. Phys. 51 (1969) 924.

13 T. Odijk, Physica A, in press.

14 G.V. Ramanathan, J. Chem. Phys. 78 (1983) 3223.

15 S. Ozeki and S. Ikeda, J. Coll. Int. Sci. 87 (1982) 424.

16 S. Ikeda, S. Ozeki and M. Tsunoda, J. Colloid Interface Sci 73 (1980) 27.

17 T. Imae, R. Kamiya and S. Ikeda, J. Colloid Interface Sci. 108 (1985) 215.

18 T. Imae and S. Ikeda, J. Phys. Chem. 90 (1986) 5216.

19 D. Stigter, Biopolymers 16 (1977) 1435.

20 T. Odijk, J. Chem. Phys. 93 (1990) 5172.

21 W.E. McMullen, W.M. Gelbart and A. Ben-Shaul, J, Chem. Phys. 85 (1986) 1088.

22 S.A. Safran, P.A. Pincus, M.E. Cates and F.C. Mac Kintosh, J. Physique (France) 51 (1990) 503.

23 F.C. MacKintosh, S.A. Safran and P.A. Pincus, Europhys. Lett. 12 (1990) 697.

24 W.M. Gelbart, A. Ben-Shaul, W.E. McMullen and A. Masters, J. Phys. Chem. 88 (1984) 861. 